\title{
8 states triple binary convolutional encoders for the construction of turbo codes
}

\author{
Horia Balta ${ }^{1, a}$ and Maria Balta ${ }^{1, b}$ \\ ${ }^{1}$ University Politehnica of Timişoara, 300223, Romania \\ ahoria.balta@etc.upt.ro (corresponding author), ${ }^{b}$ maria_lucia15@yahoo.de
}

Keywords: channel coding, convolutional code, encoding, digital communications, turbo code.

\begin{abstract}
This paper presents the rate 3/4 (triple-binary) memory 3 recursive and systematic convolutional encoders with a single shift register (TBEm3) implemented in the observer canonical form with the best frame error rate (FER) versus signal to noise ratio (SNR) performance in configuration turbo (parallel concatenated) The triple-binary turbo-codes (TBTC) were compared in terms of performance with the turbo-codes (TC) from the actual standards of communications, at the same turbo-coding rates of $3 / 5$ and 3/4. Besides the very good FER/SNR performance, the TBTC present several other advantages that recommend them: an effect ,error floor” low, latency diminution, more compact blocks - lower delay caused by interleaving, the possibility to connect the encoder TBTC to modulation blocks of higher order.
\end{abstract}

\section{Introduction}

This paper proposes the use of the TBTC, whose natural rate is $3 / 5$. The component convolutional codes for these TC have three entries. The advantages of using such TC are given by a better structuring of the data block. Thus, for TBTC, the data block is more compact in comparison with the single-binary turbo codes (SBTC) or to those duo-binary turbo codes (DBTC). Among the benefits of this compact structure are: reducing delay caused by the interleaving blocks, latency diminution, direct connection possibility to modulation blocks of higher order, possibility of intrasymbol interleaving. In addition, the simulations made with TBTC show that they are comparable in performance with the SBTC used in 3GPP, [1] and with the DBTC used in DVB-RCS, [2], for the same memory 3 of the encoders.

The TBEm 3 family investigated in this paper has over 5000 components. The purpose of the investigations was to select, from these encoders, the ones that provide the best performance in TBTC. The selection was made through an exhaustive search based on the convergence of the iterative decoding process of the TBTC, formed with each TBEm3. The quick convergence as a selection criterion provides on one way a precision good enough to identify the best TBEm3, and on the other way, a processing speed high enough to investigate the whole set of encoders. The performed ranking, with the help of the turbo-decoding convergence on the $5000 \mathrm{TBEm} 3$ has allowed the selection and the investigation of the best 200 of them. For these selected 200 TBEm3 we performed simulations in turbo configuration and we estimated their FER/SNR performance. Finally we presented the best $10 \mathrm{TBE} 33$. The study presented in this paper will be continued through the search of interleaver pairs for these 10 selected TBEm3 in order to construct TBTC.

\section{Triple-Binary Convolutional Encoders}

Encoding matrix. The general scheme of a recursive and systematic convolutional encoder, with $R=3$ inputs and memory $M=3$, in observer canonical from, is presented in Fig. 1. Denoting the moment of current time clock by $t$, by $\mathbf{s}_{t}=\left[\begin{array}{ll}s_{t, 3} & s_{t, 2} s_{t, 1}\end{array}{ }^{*}\right.$ (where $\mathbf{A}^{*}$ is the transpose of $\mathbf{A}$ ) the state vector for the encoder at moment $t$, and with $\mathbf{u}_{t}=\left[\begin{array}{ll}u_{t, 3} & u_{t, 2} \\ u_{t, 1}\end{array}\right]^{*}$ the input vector from moment $t$, the relations for the observer canonical form are: 


$$
\left\{\begin{array}{l}
s_{t+1,3}=g_{3,3} \cdot u_{t, 3}+g_{3,2} \cdot u_{t, 2}+g_{3,1} \cdot u_{t, 1}+g_{3,0} \cdot u_{t, 0} \\
s_{t+1,2}=g_{2,3} \cdot u_{t, 3}+\ldots+g_{2,0} \cdot u_{t, 0}+s_{t, 3} \\
s_{t+1,1}=g_{1,3} \cdot u_{t, 3}+\ldots+g_{1,0} \cdot u_{t, 0}+s_{t, 2} \\
u_{t, 0}=g_{0,3} \cdot u_{t, 3}+g_{0,2} \cdot u_{t, 2}+g_{0,1} \cdot u_{t, 1}+s_{t, 1}
\end{array}\right.
$$

or in the more compact matrix form:

$$
\left[\begin{array}{c}
s_{t=1,3} \\
s_{t=1,2} \\
s_{t=1,1}
\end{array}\right]=\left[\begin{array}{lll}
g_{3,3} & g_{3,2} & g_{3,1} \\
g_{2,3} & g_{2,2} & g_{2,1} \\
g_{1,3} & g_{1,2} & g_{1,1}
\end{array}\right] \cdot\left[\begin{array}{c}
u_{t, 3} \\
u_{t, 2} \\
u_{t, 1}
\end{array}\right]+u_{t, 0} \cdot\left[\begin{array}{c}
g_{3,0} \\
g_{2,0} \\
g_{1,0}
\end{array}\right]+\left[\begin{array}{c}
0 \\
s_{t, 3} \\
s_{t, 2}
\end{array}\right] .
$$

With the denotations:

$$
\begin{aligned}
\mathbf{G} & =\left[\begin{array}{llll}
g_{3,3} & g_{3,2} & g_{3,1} & g_{3,0} \\
g_{2,3} & g_{2,2} & g_{2,1} & g_{2,0} \\
g_{1,3} & g_{1,2} & g_{1,1} & g_{1,0} \\
g_{0,3} & g_{0,2} & g_{0,1} & g_{0,0}
\end{array}\right] \text { - descriptive matrix of the implementation form (dif-matrix), } \\
\mathbf{G}_{0} & =\left[\begin{array}{lll}
g_{3,3} & g_{3,2} & g_{3,1} \\
g_{2,3} & g_{2,2} & g_{2,1} \\
g_{1,3} & g_{1,2} & g_{1,1}
\end{array}\right] \text { the core of dif-matrix, }
\end{aligned}
$$

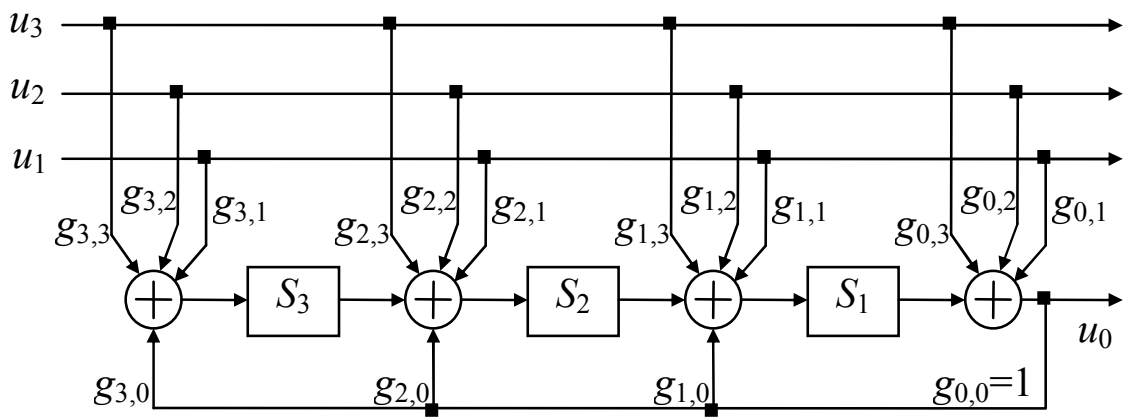

Fig. 1 Memory 3 TBC encoder in observer canonical form.

$\mathbf{G}_{F}=\left[\begin{array}{lll}g_{3,0} & g_{2,0} & g_{1,0}\end{array}\right]^{*}-$ feedback vector and

$\mathbf{G}_{L}=\left[\begin{array}{lll}g_{0,3} & g_{0,2} & g_{0,1}\end{array}\right]^{*}-$ free terms vector,

Eq. 2 can be re-written, taking into the last equation from (1), in the form:

$$
\left(\mathbf{s}_{t+1}\right)_{3 \times 1}=\left(\mathbf{G}_{T}\right)_{3 \times 3} \cdot\left(\mathbf{u}_{t+1}\right)_{3 \times 1}+(\mathbf{T})_{3 \times 3} \cdot\left(\mathbf{s}_{t}\right)_{3 \times 1},
$$

where:

$$
\mathbf{T}=\left[\begin{array}{lll}
0 & 0 & g_{3,0} \\
1 & 0 & g_{2,0} \\
0 & 1 & g_{1,0}
\end{array}\right]
$$

and:

$$
\mathbf{G}_{T}=\mathbf{G}_{0}+\mathbf{G}_{F} \cdot \mathbf{G}_{L}
$$


The last equation from (1) becomes:

$$
u_{t, 0}=\mathbf{G}_{L} \cdot \mathbf{u}_{t}+\mathbf{W} \cdot \mathbf{s}_{t},
$$

where: $\mathbf{W}=\left[\begin{array}{lll}0 & 0 & 1\end{array}\right]$. By applying the $D$ transform to Eq. 3 and Eq. 6 we obtain:

$$
\begin{aligned}
& D^{-1} \cdot \mathbf{S}(D)=\mathbf{G}_{T} \cdot \mathbf{U}(D)+\mathbf{T} \cdot \mathbf{S}(D), \\
& U_{0}(D)=\mathbf{G}_{L} \cdot \mathbf{U}(D)+\mathbf{W} \cdot \mathbf{S}(D) .
\end{aligned}
$$

The $D$-transform, [3,4], of the sequence $x_{n}$ is $X(D)=\sum_{n=-\infty}^{\infty} x_{n} \cdot D^{n}$. It can be shown that the matrix $\left(\mathbf{I}_{3}+D \cdot \mathbf{T}\right)$ is invertible, then Eq. 7 has unique solution: $\mathbf{S}(D)=D \cdot\left(\mathbf{I}_{3}+D \cdot \mathbf{T}\right)^{-1} \cdot \mathbf{G}_{T} \cdot \mathbf{U}(D)$. $\mathbf{I}_{3}$ is the unit matrix of rank 3 . The inverse of the matrix $\left(\mathbf{I}_{3}+D \cdot \mathbf{T}\right)$ is expressed as:

$$
\left(\mathbf{I}_{3}+D \cdot \mathbf{T}\right)^{-1}=\boldsymbol{\Delta}_{3}(D) \cdot\left(\frac{D}{g_{0}(D)} \cdot \mathbf{G}_{F} \cdot\left[\begin{array}{lll}
D^{2} & D & 1
\end{array}\right]+\mathbf{I}_{3}\right)
$$

where $\boldsymbol{\Delta}_{3}(D)=\left[\begin{array}{ccc}1 & 0 & 0 \\ D & 1 & 0 \\ D^{2} & D & 1\end{array}\right]$. Replacing an performing the computations, Eq. 8 becomes $U_{0}(D)=\mathbf{Q}(D) \cdot \mathbf{U}(\mathrm{D})$, with the encoding matrix, [3,4], given by:

$$
\mathbf{Q}(D)=\left[\begin{array}{lll}
\frac{g_{3}(D)}{g_{0}(D)} & \frac{g_{2}(D)}{g_{0}(D)} & \frac{g_{1}(D)}{g_{0}(D)}
\end{array}\right],
$$

where the generating polynomials are $g_{r}(D)=\sum_{m=0}^{3} g_{m, r} \cdot D^{m}, 0 \leq r \leq 3$, and $g_{0,0}=1$. Because the encoding matrix $\mathbf{Q}(D)$ given by Eq. 10 is completely identified by the dif-matrix $\mathbf{G}$, it results that there are an one-by-one correspondence ${ }^{1}$ between the set of TBEm 3 and the encoding matrices on the form (10). So, a search for an encoding matrix is equivalent with a search for a TBEm3, defined by its dif-matrix $\mathbf{G}$. For an easy identification of the matrix $\mathbf{G}$, we will accept in the following the formal identity:

$$
\mathbf{G}=\left[g_{3} g_{2} g_{1} g_{0}\right]_{10}
$$

where $g_{r}, 0 \leq r \leq 3$, represents the conversion in decimal of the binary sequence of the polynomial coefficients $g_{r}(D)$. Since we assumed that the memory encoders is $M=3$, the coefficients $g_{3}, g_{2}, g_{1}$, and $g_{0}$ are numbers between 1 and $2^{M+1}-1=15$.

The TBEm3 set cardinality. In this sub-section we will make some references to the cardinality of the TBEm3 set, having the form (11), which are the candidates for TBTC. To increase the efficiency of the search, we will impose, therefore, some restrictions:

i) $-g_{i}>0$, the 0 value would cancel the effect of that input,

ii) $-g_{0}$ must be an odd number to respect the condition $g_{0,0}=1$,

iii) - it is sufficient to consider $g_{3} \geq g_{2} \geq g_{1}$ because the change of the two entries does not practically lead to another encoder (at least in terms of performance),

$i v)$ - to define an encoder of memory $M$, it is necessary that $\max \left(g_{3}, g_{2}, g_{1}, g_{0}\right) \geq 2^{M}=H$,

The number of dif-matrices $\mathbf{G}$ of form (11) that satisfy the conditions $i$ )-iii) is:

$$
N_{i i i}(H)=H \cdot \sum_{j=1}^{2 \cdot H-1} \sum_{i=1}^{j} i=(2 \cdot H)^{2} \cdot\left[(2 \cdot H)^{2}-1\right] / 12,
$$

and those that also satisfy $i v)$ is $N_{i v}(H)=N_{i i i}(H)-N_{i i i}(H / 2)$. If we customize for $H=2^{M}=8$, we have $N_{i v}(8)=5104$.

\footnotetext{
1.e. there could be not two different TMEm3 with the same encoding matrix;
} 
An additional condition for the $\mathbf{G}$ dif-matrix could be:

$v)$ - the dif-matrix $\mathbf{G}$ must be maximum rank.

This condition is not strictly necessary ${ }^{2}$ for the decodable code, [4], but generally the performance codes satisfy it. The encoders set cardinality that also satisfies $v$ ) is $N_{v}(8)=1792$. For completeness the investigations presented in the following had in view the $N_{i v}(8)$ encoders set.

Another aspect of the search of TBEm3 is the equivalence, in terms of performance (FER/SNR) of some encoder pairs (dif-matrices).

Thus in [5] we shown that two encoders, given by the dif-matrices $\mathbf{G}=\left[\begin{array}{llll}g_{3} & g_{2} & g_{1} & g_{0}\end{array}\right]$ and $\mathbf{G}_{\mathbf{M}}=\left[\mathrm{M}\left(g_{3}\right) \mathrm{M}\left(g_{2}\right) \mathrm{M}\left(g_{1}\right) \mathrm{M}\left(g_{0}\right)\right]$ are equivalent in performance. $\mathrm{M}(x)$ is the mirror image of $x$. So if $x=13=1101_{2}$, then $\mathrm{M}(x)=1011_{2}=11$. Considering this equivalence in performance in the search of the dif-matrices of the TBEm3, from a $\mathbf{G}$ and $\mathbf{G}_{\mathrm{M}}$, we just kept $\mathbf{G}$ in the investigated set. Thus we reduced the search efforts.

\section{Search on the TBEm3 Set}

In this chapter we present the TBEm3 identified by dif-matrices (11), with the best performances in TBTC and the algorithmic steps that led to their selection.

The parameters of the TBTC used in simulations. We will start by presenting the TC parameters from the simulations. We used a parallel concatenation of the investigated component codes, [6]. For the trellis closing we used the tail-biting technique (circular, [7]). The interleaving was realized on two levels: intra- and inter-symbol. The intra-symbol interleaving operates on the three bits $u_{t, 3}$, $u_{t, 2}$ and $u_{t, 1}$ from the symbol $u_{t}, 0 \leq t<N$, where $N$ is the number of symbols from a data block (block length). The intra-symbol interleaving is suggested by Fig. 2. For the inter-symbol interleaving we used either an S-type interleaver, [8,9], with $S=23$, or a QPP type interleaver, [10], with the length $N=500$ and the defining parameters $f_{1}=9$ and $f_{2}=50$. We considered an AWGN (Additive White Gaussian Noise) channel and we used a BPSK (Binary Phase Shift Keying) modulation. For decoding we used the MaxLogMAP algorithm, [11], a maximum number of 15 iterations and a stop criterion defined in [12].

The search results on the whole set of encoders. For each $\mathbf{G}_{n}$ dif-matrix, $1 \leq n \leq 5104$, on the form (11), we built a TBTC with the parameters defined before. We performed simulations with these TBTC. Thus we obtained a $C_{v}$ value of the convergence associated to the $\mathbf{G}_{n}$ dif-matrix, where:

$$
C_{v}=\lim _{N b \rightarrow \infty} \frac{i t e r(N b)}{N b} .
$$

The information block before the intra-symbol interleaving:

\begin{tabular}{|l|l|l|l|l|l|l|l|l|l|}
\hline$u_{0,2}$ & $u_{1,2}$ & $u_{2,2}$ & $u_{3,2}$ & $u_{4,2}$ & $u_{5,2}$ & $u_{6,2}$ & $u_{7,2}$ & $u_{8,2}$ & $u_{9,2}$ \\
\hline$u_{0,1}$ & $u_{1,1}$ & $u_{2,1}$ & $u_{3,1}$ & $u_{4,1}$ & $u_{5,1}$ & $u_{6,1}$ & $u_{7,1}$ & $u_{8,1}$ & $u_{9,1}$ \\
\hline$u_{0,0}$ & $u_{1,0}$ & $u_{2,0}$ & $u_{3,0}$ & $u_{4,0}$ & $u_{5,0}$ & $u_{6,0}$ & $u_{7,0}$ & $u_{8,0}$ & $u_{9,0}$ \\
\hline
\end{tabular}

The information block after the intra-symbol interleaving:

\begin{tabular}{|l|l|l|l|l|l|l|l|l|l|}
\hline$u_{0,2}$ & $u_{1,1}$ & $u_{2,0}$ & $u_{3,2}$ & $u_{4,1}$ & $u_{5,0}$ & $u_{6,2}$ & $u_{7,1}$ & & $u_{9,2}$ \\
\hline$u_{0,1}$ & $u_{1,0}$ & $u_{2,2}$ & $u_{3,1}$ & $u_{4,0}$ & $u_{5,2}$ & $u_{6,1}$ & $u_{7,0}$ & $u_{8,2}$ & $u_{9,1}$ \\
\hline$u_{0,0}$ & $u_{1,2}$ & $u_{2,1}$ & $u_{3,0}$ & $u_{4,2}$ & $u_{5,1}$ & $u_{6,0}$ & $u_{7,2}$ & $u_{8,1}$ & $u_{9,0}$ \\
\hline
\end{tabular}

Fig. 2 Intra-symbol interleaving. Example for $N=10$.

\footnotetext{
${ }^{2}$ because we considered only systematic codes. (To have decodable codes such a condition has to be imposed to the complete generator matrix, which is $\left[\mathbf{I}_{R} \mathbf{Q}^{*}\right]$, but because $\mathbf{I}_{R}$ is the unit matrix of rank $R$, the condition is assured regardless $\mathbf{Q}$ or $\mathbf{G}$ respectively.)
} 
Table I. The selected best matrices

\begin{tabular}{|c|c|c|c|}
\hline No & Initial rank & G & $\mathbf{G}_{\text {शr }}$ \\
\hline 1 & 7 & $\begin{array}{llll}13 & 9 & 3 & 11\end{array}$ & $1211 \quad 913$ \\
\hline 2 & 4 & $\begin{array}{lllll}15 & 14 & 11 & 13\end{array}$ & $1513 \quad 711$ \\
\hline 3 & 2 & $\begin{array}{llll}13 & 9 & 711\end{array}$ & 1411913 \\
\hline 4 & 1 & $1310 \quad 911$ & 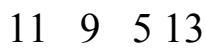 \\
\hline 5 & 18 & $1511 \quad 513$ & $1513 \quad 1011$ \\
\hline 6 & 6 & $\begin{array}{llll}15 & 9 & 713\end{array}$ & $1514 \quad 911$ \\
\hline 7 & 50 & $1511 \quad 113$ & $1513 \quad 811$ \\
\hline 8 & 11 & $\begin{array}{llll}13 & 7 & 4 & 11\end{array}$ & 1411 \\
\hline 9 & 17 & $1310 \quad 311$ & $1211 \quad 513$ \\
\hline 10 & 15 & $1510 \quad 913$ & 159 \\
\hline
\end{tabular}

Table II. The tc's parameters used in simulations

\begin{tabular}{|c|c|c|c|}
\hline & SBTC & DBTC & TBTC \\
\hline $\begin{array}{l}\text { Component code } \\
\text { generator matrix }\end{array}$ & $\mathbf{G}=\left[\begin{array}{ll}11 & 13\end{array}\right]$ & $\mathbf{G}=\left[\begin{array}{lll}13 & 15 & 11\end{array}\right]$ & $\mathbf{G}=\left[\begin{array}{lllll}13 & 9 & 3 & 1\end{array}\right]$ \\
\hline Closure of the trellis & $\begin{array}{l}\text { uninterleaved dual } \\
\text { termination }\end{array}$ & circular, (tail biting) & circular, (tail biting) \\
\hline $\begin{array}{l}\text { Data block } \\
\text { dimension }(R \times N)\end{array}$ & $1 \times 1504=1504$ bits & $2 \times 752=1504$ bits & $3 \times 500=1500$ bits \\
\hline Coding rate, $R_{c}$ & $3 / 5 \quad 3 / 4$ & $3 / 5 \quad 3 / 4$ & $3 / 5 \quad 3 / 4$ \\
\hline $\begin{array}{l}\text { Puncturing matrices } \\
\text { related to the coding } \\
\text { rates }\end{array}$ & $\begin{array}{c}\quad\left[\begin{array}{lll}1 & 0 & 0 \\
0 & 1 & 0\end{array}\right], \\
{\left[\begin{array}{llllll}1 & 0 & 0 & 0 & 0 & 0 \\
0 & 0 & 0 & 1 & 0 & 0\end{array}\right]}\end{array}$ & $\begin{array}{l}{\left[\begin{array}{lll}1 & 1 & 0 \\
0 & 1 & 1\end{array}\right],} \\
{\left[\begin{array}{lll}1 & 0 & 0 \\
0 & 1 & 0\end{array}\right]}\end{array}$ &,$-\left[\begin{array}{ll}1 & 0 \\
0 & 1\end{array}\right]$ \\
\hline $\begin{array}{l}\text { inter-symbol } \\
\text { interleaving }\end{array}$ & $\begin{array}{c}\text { Interleaver QPP } \\
\text { defined in [1] with } \\
f_{1}=49, f_{2}=846\end{array}$ & $\begin{array}{c}\text { Interleaver ARP } \\
\text { defined in [2] with } \\
P=19, P_{1}=376, \\
P_{2}=224, P_{3}=600\end{array}$ & $\begin{array}{c}\text { Interleaver QPP } \\
\text { defined in [1] with } \\
\quad f_{1}=9, f_{2}=50\end{array}$ \\
\hline memory; no. inputs & $3 ; 1$ & $3 ; 2$ & $3 ; 3$ \\
\hline trellis states / tact & 8 & 8 & 8 \\
\hline no. branchs / state & 2 & 4 & 8 \\
\hline trellis branchs / tact & 16 & 32 & 64 \\
\hline No. tacts & 1504 & 752 & 500 \\
\hline Total branchs & $16 \times 1504=24,064$ & $32 \times 752=24,064$ & $64 \times 500=32,000$ \\
\hline $\begin{array}{l}\text { Forward/backward } \\
\text { coefficients }\end{array}$ & $8 \times 1504=12,032$ & $8 \times 752=6016$ & $8 \times 500=4000$ \\
\hline $\begin{array}{l}\text { Extrinsic information } \\
\text { coefficients }\end{array}$ & 1504 & $4 \times 752=3008$ & $8 \times 500=4000$ \\
\hline $\begin{array}{l}\text { Simulation time for } \\
10.000 \text { blocks }(\mathrm{sec})\end{array}$ & 117 & 114 & 139 \\
\hline
\end{tabular}

We order the set of the 5,104 encoders after $C_{v}$. We received the function $C_{v}: J \rightarrow[1,15]$, where $J=\{1,2, \ldots 5104\}$. The simulations were performed at a signal to noise ratio (SNR) of $4 \mathrm{~dB}$ with an $\mathrm{S}$ type interleaver with $N=500$. For the second step of the selection we retained 200 encoders having the lowest convergence. 
To avoid including (through the 200) of both matrices from a pair of mirror equivalent matrices, we did as follows. We selected the fist matrix, $\mathbf{G}_{1}$, with $C_{v}(1)=$ minimum. We searched for its mirror equivalent matrix, $\mathbf{G}_{1 \mathrm{M}}$. We removed the $\mathbf{G}_{1}$ and $\mathbf{G}_{1 \mathrm{M}}$ matrices from the search basis. We selected $\mathbf{G}_{2}$, with the lowest convergence, eliminating $\mathbf{G}_{2}$ and $\mathbf{G}_{2 \mathrm{M}}$ from the search basis, and so on. We note here that the simulations confirmed that the TBEm 3 defined by the pairs $\mathbf{G}_{i}$ and $\mathbf{G}_{\mathrm{iM}}$ have similar performance.

In the second step, for the 200 matrices, we simulated the transmission of $2 \cdot 10^{7}$ blocks with the corresponding TBTC, calculating FER and bit error rate (BER). The simulations where performed at a $S N R=4 \mathrm{~dB}$ and a random $\mathrm{S}$ type interleaver with $S=23$. (We used this interleaver in the first step, because being a uniform interleaver is more "impartial" in the encoders appreciation.)

In Table I was shown the TBEm3s with the best FER performance and also their mirror equivalents. (The columns of $\mathbf{G}_{\mathrm{M}}$ were obtained from the columns of $\mathbf{G}$ through a mirroring and a reordering.)

The TBTC performance versus SBTC and DBTC. In this sub-section we compare the performance of the TBTC that has like a component encoder the TBEm3 defined by the dif-matrix $\mathbf{G}_{7}=\left[\begin{array}{llll}13 & 9 & 3 & 11\end{array}\right]$, with the performance of the SBTC defined in 3GPP standard, [1], and with the DBTC defined in DVB-RCS standard, [2]. For an accurate comparison, we used, for all TC, the same encoding rates, $R_{c}=0.60$, respectively $R_{c}=0.75$. Also we tried to use the same number for the input data blocks. As so, for SBTC we used blocks with the size of $1 \times 1504$, respectively the QPP interleaver having $f_{1}=49$ and $f_{2}=846$ (see Table 5.1.3-3, [1] page 14). For DBTC, the blocks had the size $2 \times 752$, and we used the interleaving described in [2], page 24 . We considered an AWGN channel and a BPSK modulation. We used the magic genie criterion [13] to stop the iterations. For the non-stopped blocks we simulate a maximum number of iteration iter $_{\max }=8$.

In order to obtain the desired coding rates we used puncturing. The parameters described above and the puncturing matrices (the zeros indicate the positions of the deleted bits from the redundant sequences of the TC) are shown in Table II. Also in Table II we have shown some details about the trellis structure of the three TC, in order to evaluate the decoding complexity. Thus, for each iterations the TBTC has to calculate a number of 32,000 branch metrics, $2 \times 4000$ forward and backward coefficients and 4000 coefficients of extrinsic information.

In comparison the SBTC has to calculate $24,064 / 2 \times 12,032 / 1504$ coefficients and the DBTC $24,064 / 2 \times 6016 / 3008$ coefficients. Obviously this is a rough assessment because the coefficients calculations differ from a TC type to another. A fairer assessment is the average processing time of a block. In the last line of Table II we indicated the required time to process 10,000 blocks. TBTC works $19 \%$ more for a block than SBTC and 22\% more than the DBTC. The simulation results are presented in the diagrams in Fig. 3 and Fig. 4. We have simulated until we obtain 500 erroneous blocks but no more than $10^{9}$ transmitted blocks. 


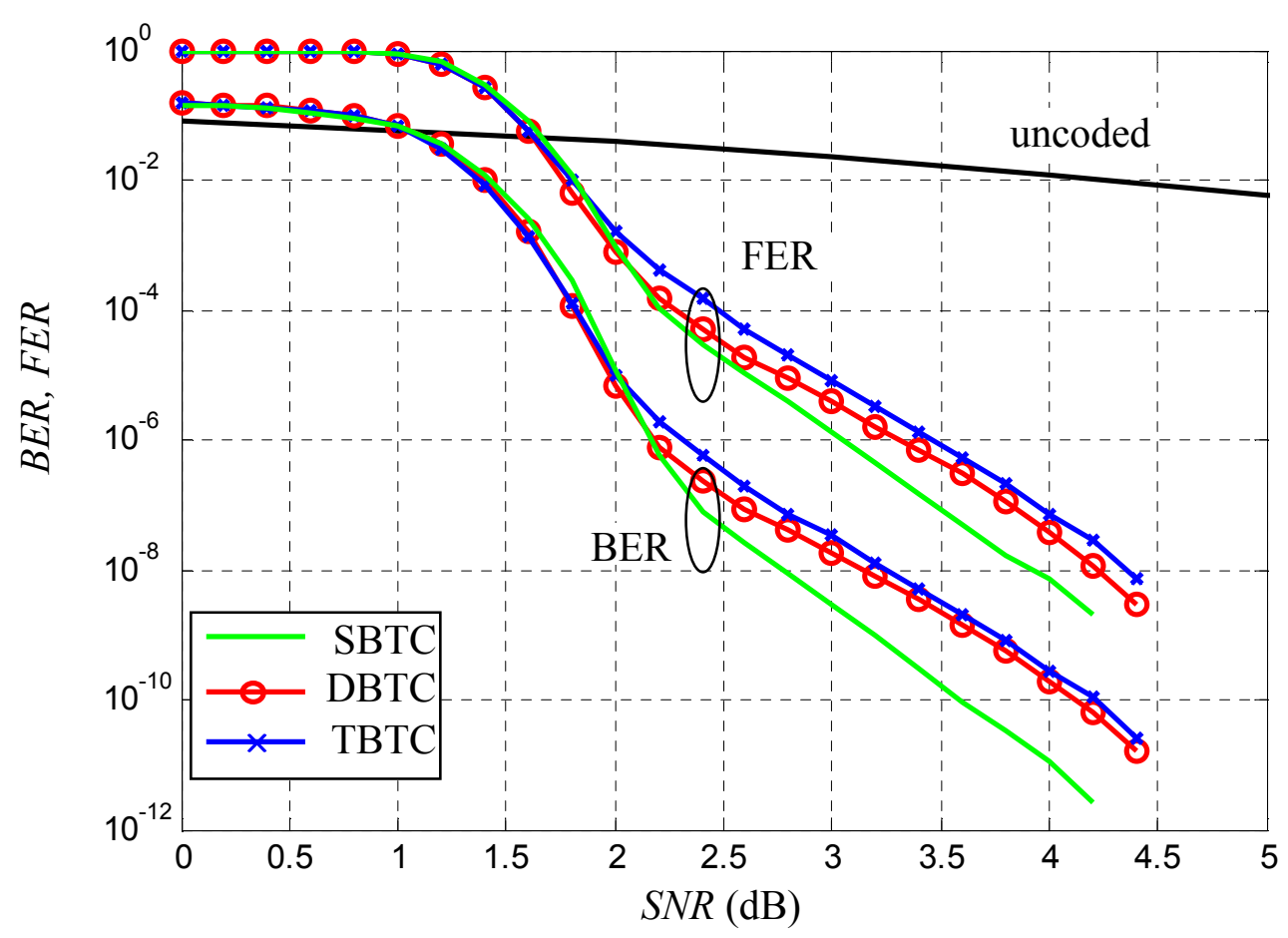

Fig. 3 Performance comparison between SBTC, DBTC and TBTC at coding rate $R_{c}=0.60$

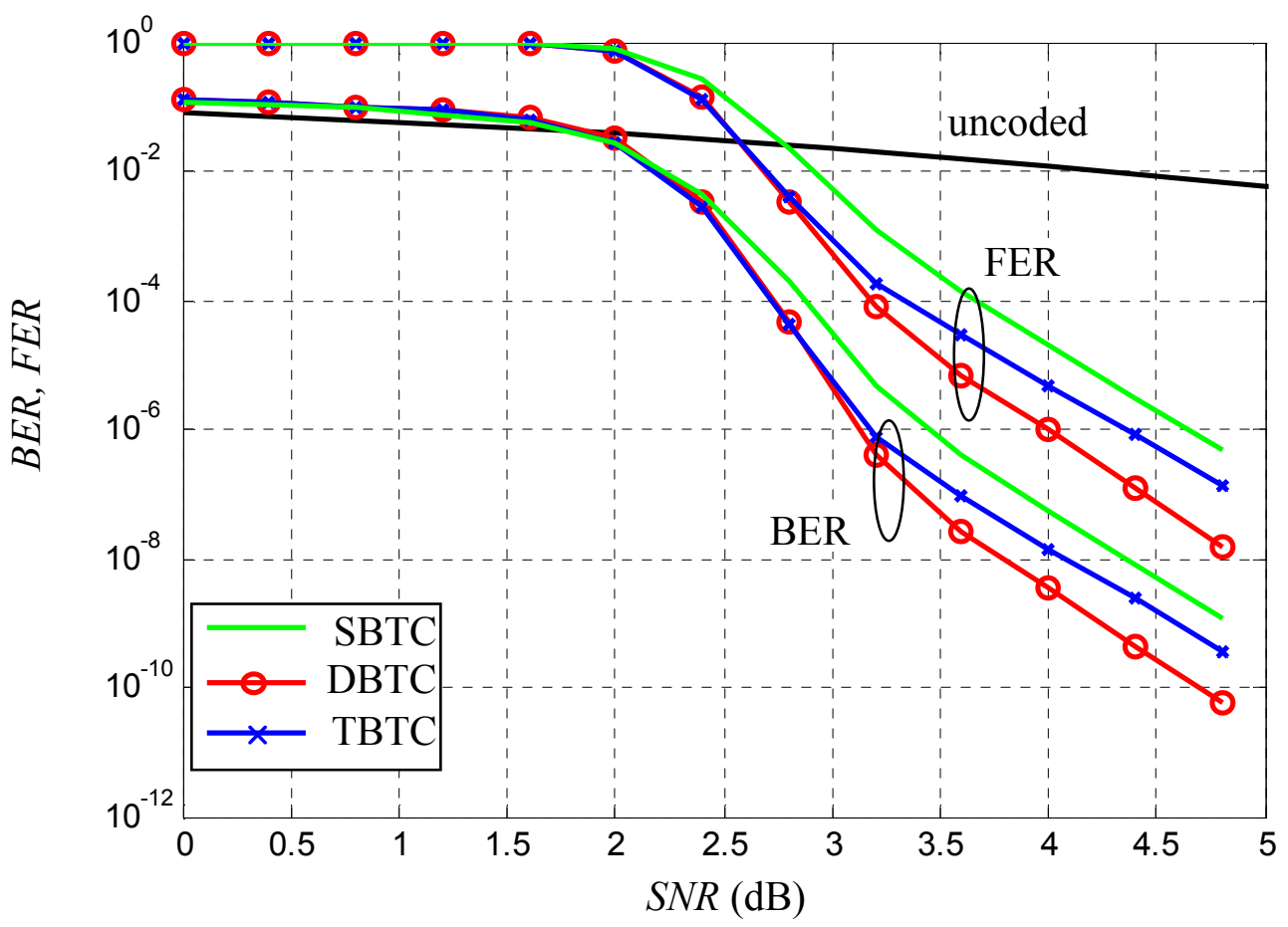

Fig. 4 Performance comparison between SBTC, DBTC and TBTC at coding rate $R_{c}=0.75$

We used a $0.2 \mathrm{~dB}$ step in SNR for $R_{c}=0.60$ (Fig. 3) and a $0.4 \mathrm{~dB}$ SNR step for the coding rate $R_{c}=0.75$ (Fig. 4 ). We can see that the performance of the three TC depends on the coding rate. So, if for $R_{c}=0.60 \mathrm{SBTC}$ is superior to the others, at $R_{c}=0.75$ the best one is DBTC. The performance of TBTC is similar to DBTC at $R_{c}=0.60$, but inferior to SBTC. However, if we choose iter $_{\text {max }}=3$, the performance of the TBTC overcame the SBTC one. This mean that the TBTC is faster convergent that the SBTC. At $R_{c}=0.75$, the performance of the TBTC is worst than the performance of DBTC but better than the SBTC one. 


\section{Conclusion}

The bit error rate and frame error rate performance of triple binary turbo code that incorporate the recursive and systematic triple-binary convolutional encoders of memory 3 (8 states) and natural rate of $3 / 4$ is close to the performance of the single binary turbo code of the 3GPP standard and of the double binary turbo code of the DVB-RCS standard. However, triple binary turbo code offer the advantage of a better (more compact) structure of the data blocks. A possible improvement of the TBTC performance could be given by an intra-symbol interleaving correlated with the inter-symbol interleaving. These might be the subject of a future study.

\section{Acknowledgment:}

This paper was supported by the project "Development and support of multidisciplinary postdoctoral programmes in major technical areas of national strategy of Research - Development Innovation" 4D-POSTDOC, contract no. POSDRU/89/1.5/S/52603, project co-funded by the European Social Fund through Sectoral Operational Programme Human Resources Development 2007-2013.

\section{References}

[1] 3rd Generation Partnership Project; Technical Specification Group Radio Access Network; Evolved Universal Terrestrial Radio Access (E-UTRA); document 10.2.0 (2011-06) from: http://www.3gpp.org/ftp/Specs/html-info/36212.htm

[2] European Telecommunications Standards Institute., Interaction channel for satellite distribution systems. $\quad$ V1.3.1 $301 \quad 790, \quad$ ETSI EN, March 2003, http://www.etsi.org/deliver/etsi_en/301700_301799/301790/01.03.01_60/en_301790v010301p.pdf.

[3]R. Johannesson, and Z. Wan, "A Linear Algebra Approach to Minimal Convolutional Encoders", IEEE T Inform Theory, 39 (1993) 1219-1233.

[4]R. Johannesson, K. Sh. Zigangirov, Fundamentals of Convolutional Coding, IEEE Press, 1999.

[5]H. Balta, A. Isar, D. Isar, M. Balta, Mirror equivalent turbo-codes - part I, ICCEN, Hong Kong, Nov. 25-27, (2011) 25-31.

[6]C. Berrou, A. Glavieux, P. Thitimajshima, Near Shannon Limit Error -Correcting Coding and Decoding: Turbo -Codes, Proc. of ICC, Geneve, May 23-26, 2 (1993) 1064-1070.

[7]C. Weiss, C. Bettstetter, S. Riedel, and D. J. Costello, Turbo decoding with tailbiting trellises, Proc. IEEE Int. Symp. Signals, Syst., Electron., Pisa, Italy, Oct. (1998) 343-348.

[8]D. Divsalar and F. Pollara, “Multiple Turbo Codes”, MILCOM'95, Nov. 6-8, (1995) 279-285.

[9]S.N. Crozier, „New High-Spread High-Distance Interleavers for Turbo-Codes”, Proc. 20th Biennial Symp. Commun., Kingston, Canada, (May. 2000) 3-7.

[10] Q J. Sun and O. Y. Takeshita, "Interleavers for turbo codes using permutation polynomials over integer rings," IEEE T Inform Theory, 51 (2005) 101-119.

[11] W. Koch, and A. Baier, Optimum and sub-optimum detection of coded data disturbed by time-varying intersymbol interference, GLOBECOM, (1990) 1679-1684.

[12] H. Balta, C. Douillard, M. Kovaci, The Minimum Likelihood APP Based Early Stopping Criterion for Multi-Binary Turbo Codes, Scientific Bulletin of Politehnica University of Timisoara, Transactions on Electronics and Communications, Tome 51-65, Beam 2, Timisoara, Romania, (2006) 199-203.

[13] A. Matache, S. Dolinar, and F. Pollara, Stopping rules for Turbo Decoders, JPL TMO Progress Report 42-142, August 15, (2000) 1-22. 